\title{
Analysis of the oxygen scattering behaviour on ionomer surface in catalyst layer of PEFC
}

\author{
Masataka Nakauchi ${ }^{1}$, Takuya Mabuchi ${ }^{1}$, Ikuya Kinefuchi ${ }^{2}$,Hideki Takeuchi ${ }^{3}$ and Takashi Tokumasu ${ }^{4}$ \\ ${ }^{1}$ Graduate School of Engineering, Tohoku University \\ 980-8577, Sendai, Miyagi (Japan)
}

Phone/Fax number:+81 22217 5247, e-mail: nakauchi@nanoint.ifs.tohoku.ac.jp, mabuchi@ nanoint.ifs.tohoku.ac.jp

2 Department of Mechanical Engineering, The University of Tokyo Phone/Fax number:+813 5841 6337, email: kine@fel.t.u-tokyo.ac.jp

${ }^{3}$ Department of Mechanical Engineering, National Institute of Technology, Kochi College Phone/Fax number:+818 864 5538, email: takeuchi@me.kochi-ct.ac.jp

${ }^{4}$ Institute of Fluid Science, Tohoku University Phone/Fax number:+81 22217 5239, email: tokumasu@ifs.tohoku.ac.jp

\begin{abstract}
Mass transport significantly affects the reaction efficiency of polymer electrolyte fuel cells. In particular, the oxygen transport in catalyst layers is important for the improvement of its efficiency. However, the mechanism of oxygen scattering on ionomer surface, which is one of the dominant factors of transport phenomena, has not been clarified. Therefore, we analyzed the oxygen scattering behaviour on ionomer surface using molecular dynamics simulation. Oxygen molecules are impinged to ionomer surface with different incident energies and angles. According to the total energy of oxygen molecule, the trajectories of oxygen molecules are classified into trapping or scattering. The trapping probability of oxygen molecule on ionomer surface decreases as the normal component of the incident energy increases. Oxygen molecules with low normal incident energy get energy during the gas-surface interaction on the surface and desorb from the surface. The number of collisions with the surface does not affect the energy transfer between oxygen molecule and ionomer surface.
\end{abstract}

\section{Key words}

Polymer Electrolyte Fuel Cell, Rarefied Gas Dynamics, Molecular Dynamics Simulation, Scattering Behaviour

\section{Introduction}

Polymer electrolyte fuel cells (PEFCs) have received much attention in recent years as next generation power sources. Because of their high power density and low operation temperature, PEFCs are promising power sources for stationary use, vehicles, and emergency purpose. The familiarization of PEFCs requires cost reduction, improved durability, and higher reaction efficiency. The reaction efficiency of PEFCs in high power density operation drops due to restricted mass transport in PEFCs. In particular, oxygen transport in catalyst layers (CLs) is one of the dominant causes of power losses [1]. The reduction of oxygen transport resistance is important for improving the reaction efficiency of the system.

In cathode side of PEFCs, oxygen passes thorough gas diffusion layers (GDLs), micro porous layers (MPLs), and CLs, and finally permeates ionomer thin film, which is composed of perfluorosulfonic acid membranes such as Nafion, water molecules, and hydronium ions. GDLs and MPLs are composed of micro porous carbons. In CLs, Pt catalysts are located on micro porous carbons and they are covered with ionomer. Therefore, there are two components of oxygen transport in CLs, i.e., gas transport in porous layers and permeation through ionomers. In order to accurately analyze the former transport component, Kinefuchi et al. investigated the oxygen diffusion resistance in MPLs and in CLs using the direct simulation Monte Carlo (DSMC) method [2, 3]. The numerical simulation well reproduces the experimental results in MPLs [2]. However, as shown in Figure 1, the results of the DSMC calculations, which adopt the same reflection model as for in MPLs, differ from that of experimental results in CLs [3]. In these calculations, the same reflection model is adopted for the scattering of oxygen molecules on solid surface regardless of the presence or absence of ionomer on micro porous carbons. The results of DSMC calculations depend on the scattering model at surface. Hence, the disagreement between DSMC calculations and the experimental results suggests that the oxygen scattering model cannot reproduce the real scattering phenomena on ionomer surface. The accurate analysis of oxygen transport in CLs needs a scattering model which can reproduce the scattering phenomena on ionomer surface. 


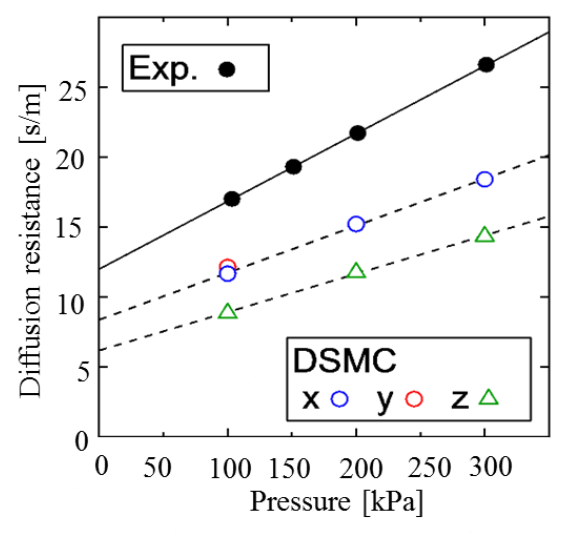

Fig.1 Diffusion resistance of oxygen in catalyst layer.

The understanding of the gas-surface interaction is important to construct of a scattering model at surface. One effective method for the gas-surface interaction analysis is the molecular dynamics (MD) simulation. We employ the MD simulation to investigate the dynamics of scattering and trapping processes of oxygen molecule on ionomer surface. In this study, we constructed a simulation system which assumed as the structure in CLs, and oxygen molecules are impinged to the ionomer surface at different incident energies and angles. The results of this study provide the trapping probability of incident molecules on ionomer surface, the number of collisions with ionomer surface, and energy loss due to the scattered oxygen molecule.

\section{Simulation Method}

In the simulation system, an ionomer surface composed of Nafion, water molecules, and hydronium ions was placed on carbon layers as shown in Figure 2. The structure of Nafion with an equivalent weight $\sim 1100$ is shown in Figure 3. 240 carbon atoms per layer were set on the bottom of the simulation domain which is $25.5 \times 24.5 \times 100 \AA^{3}$ in size. Two polymer chains and solvent molecules were put at random on the carbon layers. The water content, which indicates the ratio of the number of water molecules and hydronium ions to that of $\mathrm{SO}_{3}^{-}$, was set at 3 . The periodic boundary conditions were imposed in the $x$ and $y$ directions. Then, annealing process was applied at four times to equilibrate the system.

The DREIDING force field [4] was used for intramolecular and intermolecular potential for polymer chains. Hydronium ions and water molecules were described by the F3C model [5], and the intramolecular interaction between oxygen atoms of an oxygen molecule was represented by the Morse potential [6]. A combination of Lennard-Jones (LJ) potential and Morse potential was adopted as intermolecular potential between an oxygen molecule and solvent molecules [7, 8]. The parameters of LJ potentials for remaining intermolecular interactions were determined with Lorentz-Berthelot mixing rules. The particle mesh Ewald method for coulomb potential was applied to all charged atoms.

The impinging oxygen molecule was initially located above the potential cutoff length $(12 \AA)$ from the topmost ionomer

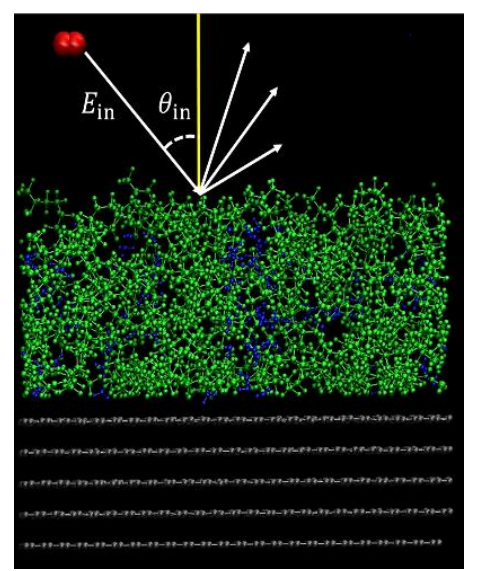

Fig.2 Simulation system of the carbon surface covered with ionomer.

(Gray: Carbon, Green: Polymer, Blue: Solvent)

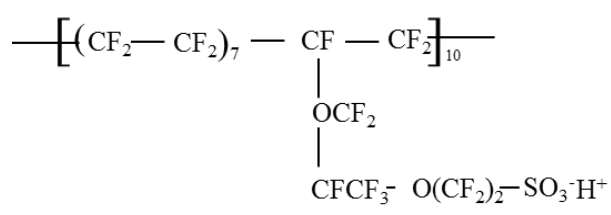

Fig.3 Structure of Nafion.

surface atom. The initial $x$ and $y$ positions of oxygen molecule were decided randomly. For initial condition, the incident transitional energy $E_{\text {in }}$ was given as $3 / 2 k_{\mathrm{B}} T_{\text {in }}$, where $T_{\text {in }}$ ranges from 150 to $600 \mathrm{~K}$ for every $150 \mathrm{~K}$. The incident angles $\theta_{\text {in }}$, which is measured from the surface normal were $0^{\circ}, 30^{\circ}$ and $60^{\circ}$. While initial energies and angles are constant, the initial orientation was given isotropically.

Simulations of 100 trajectories were performed for each set of initial conditions. Each trajectory was classified as scattered or trapped on the surface as follows. If an oxygen molecule scattered from the surface and desorbed to the height greater than the cutoff length, it was classified as scattered. If the total energy of oxygen molecule fell below $2 k_{\mathrm{B}} T_{\mathrm{S}}$, where $T_{\mathrm{S}}$ is the surface temperature, it was classified as trapped on ionomer surface [9]. Trajectory calculations were continued until the maximum time step of 20 ps was reached, or an oxygen molecule was classified as scattered or trapped.

\section{Results and Discussion}

\section{A. Trapping probability}

Trapping is important to understand gas-surface interaction. An oxygen molecule is considered to be trapped when the total energy falls below a particular value during interaction on the surface on the surface. The trapping probability is calculated as the ratio of the number of trapped molecules to the total number of trajectories. Figure 4 shows trapping probabilities of oxygen molecules on ionomer surface for different incident angles and energies. The trapping probabilities increase as the incident angle increases or the incident energy decreases. This tendency is due to the normal energy component of 


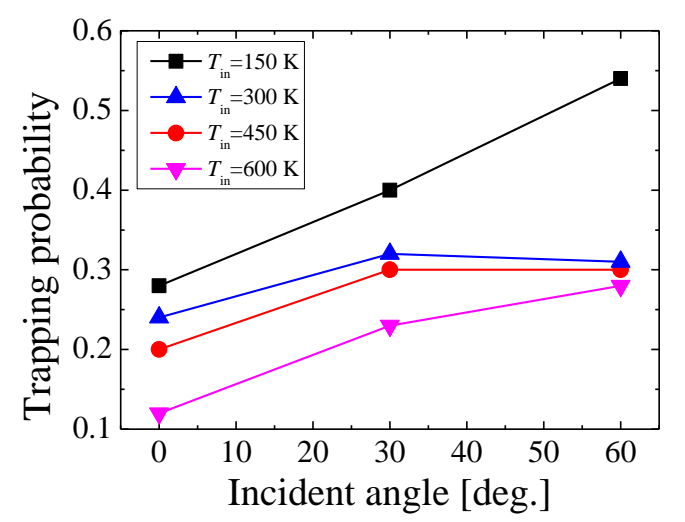

Fig.4 Trapping probability of oxygen molecule at different incident angles and incident energy.

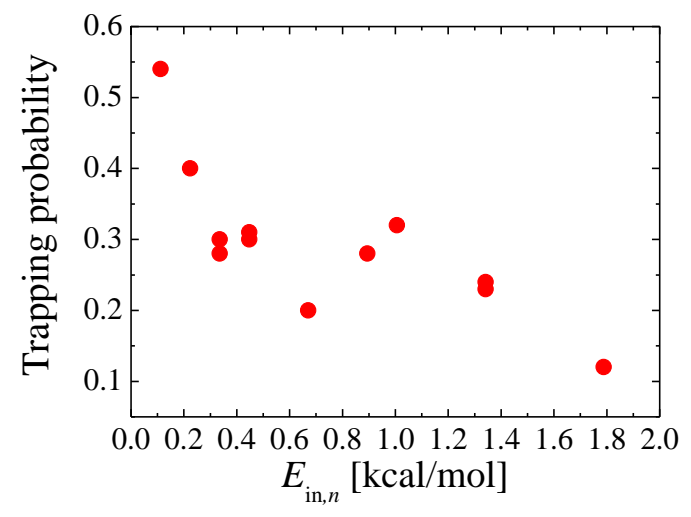

Fig.5 Trapping probability of oxygen molecule as a function of normal energy component of incident molecule.

incident molecules. The trapping probability as a function of the normal energy component of incident molecules is shown in Figure 5. $E_{\mathrm{in}, n}$ means the normal energy component of incident molecules represented by $E_{\text {in }} \cos ^{2} \theta_{\text {in. }}$. Generally, the trapping probability decreases as the normal energy component increase. Even if the incident energies are different, the trapping probabilities are well correlated with the normal energy component. These result indicate that the tangential component of incident energy does not mainly affect the trapping probability, and the normal energy component is dominant factor for the trapping probability on the surface. Oxygen molecules with high normal energy are seldom trapped on the ionomer surface.

\section{B. Energy change of a scattered oxygen molecule}

The typical example of the time history of oxygen molecule are shown in Figures 6 and 7. Figure 6 and 7 represent distance between an oxygen molecule and the closest atom of ionomer surface and the translational, rotational and potential energies of oxygen molecule in the case of $T_{\text {in }}=600 \mathrm{~K}$ or $T_{\text {in }}=150 \mathrm{~K}$, and $\theta_{\text {in }}=30^{\circ}$. These results describe how an oxygen molecule scatters at ionomer surface. Firstly, the rotational energy $E_{\text {rot }}$ and the potential energy $E_{\text {pot }}$ of the oxygen molecule is zero because the oxygen molecule has only translational energy as the incident energy and it is initially located higher than the cutoff length.

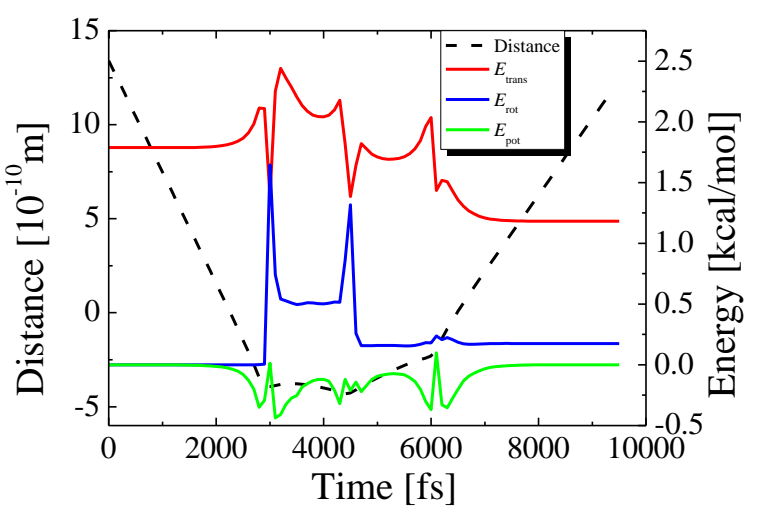

Fig.6 Time history of scatterd oxygen molecule in the case of $T_{\text {in }}=600 \mathrm{~K}$ and $\theta_{\text {in }}=30^{\circ}$.

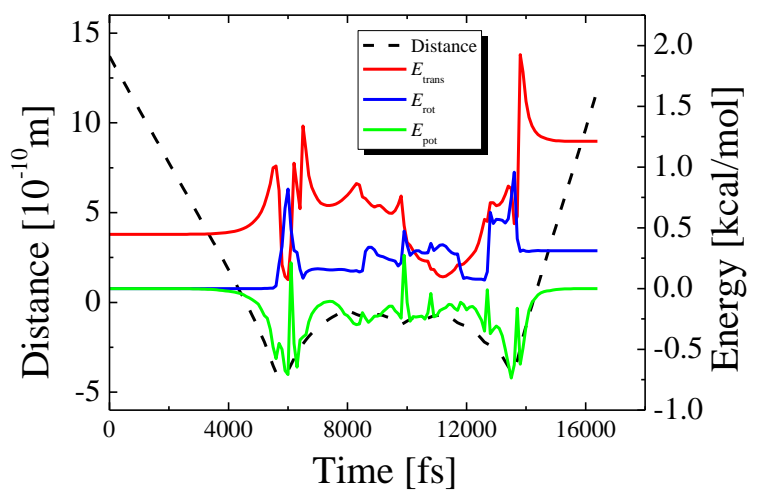

Fig.7 Time history of scatterd oxygen molecule in the case of $T_{\text {in }}=150 \mathrm{~K}$ and $\theta_{\text {in }}=30^{\circ}$.

The oxygen molecule approaches the ionomer surface, and the translational energy $E_{\text {trans }}$ is excited by the attractive force from the surface. When the oxygen molecule reaches the surface, the excitation of $E_{\text {rot }}$, which occurs by the energy transfer from translation to rotation, is observed. It also indicates the gas-surface interaction occurs the during collision with the surface. After that, the excitation of $\boldsymbol{E}_{\text {trans }}$ occurs again and oxygen molecule desorbs from the surface. In the case of $T_{\text {in }}=600 \mathrm{~K}, \boldsymbol{E}_{\text {trans }}$ decreases compared with initial condition after the gas-surface interaction. Contrary, in the case of $T_{\text {in }}=150 \mathrm{~K}, \boldsymbol{E}_{\text {trans }}$ increases after the interaction.

Two different scattering types of trajectories observed. Table I lists the ratio of the scattering type as a function of incident condition. The scattering phenomena of oxygen molecule are separated into "direct" or "nondirect" scattering. When the normal velocity component of the incident molecule changes from negative to positive, it is defined as a collision with the surface. "Direct" scattering means that an oxygen molecule collides with the surface only once while an oxygen molecule undergoes multiple collisions in "nondirect" scattering [10]. In almost all the cases, "direct" scattering accounts for more than half of the ratio. However, its ratio does not significantly change as the incident energy and angle changes. There results imply that the energy transfer does not depend on the number of collisions with the surface. 
Table I. - Ratio of scattering types

\begin{tabular}{cc|cc}
\hline \multicolumn{2}{c|}{ Initial condition } & \multicolumn{2}{c}{ Scattering type } \\
$T_{\text {in }}[\mathrm{K}]$ & $\theta_{\text {in }}[$ deg.] & direct & nondirect \\
\hline \multirow{3}{*}{150} & 0 & 0.48 & 0.52 \\
& 30 & 0.60 & 0.40 \\
& 60 & 0.59 & 0.41 \\
\hline \multirow{3}{*}{300} & 0 & 0.63 & 0.37 \\
& 30 & 0.58 & 0.42 \\
450 & 60 & 0.53 & 0.47 \\
\hline \multirow{3}{*}{600} & 0 & 0.67 & 0.33 \\
& 30 & 0.72 & 0.28 \\
& 60 & 0.68 & 0.31 \\
\hline \multirow{3}{*}{60} & 0 & 0.62 & 0.38 \\
& 30 & 0.63 & 0.39 \\
& 60 & 0.68 & 0.32
\end{tabular}

\section{Increase of translational energy of oxygen molecule}

In order to understand the energy transfer of oxygen molecule during collision, the mean increase in the translational energy increase is determined by

$$
\left\langle\Delta E_{\text {trans }}\right\rangle=\left\langle E_{f}\right\rangle-\left\langle E_{\text {in }}\right\rangle
$$

where $E_{f}$ is the translational energy of a scattered oxygen molecule. Figure 8 shows the average of translational energy as a function of the normal incident energy component. Error bars represent a standard deviation. In general, oxygen molecules loses their translational energy as the normal incident energy component increases. Contrary, oxygen molecules with low incident energy get the energy from the surface during the interaction.

\section{Conclusion}

The scattering and trapping dynamics of oxygen molecules on ionomer surface have been investigated using molecular dynamics simulation. The trapping probability of oxygen molecules on the ionomer surface is well correlated with the normal incident energy. Oxygen molecules with relatively low incident energy receive energy from the surface, and the translational energy is excited by the gas-surface interaction. In contrast, Oxygen molecules with high incident energy loses their translational energy during the interaction. The number of collisions does not significantly change, and the energy transfer between oxygen molecule and ionomer surface does not depend on the number of collisions with the surface.

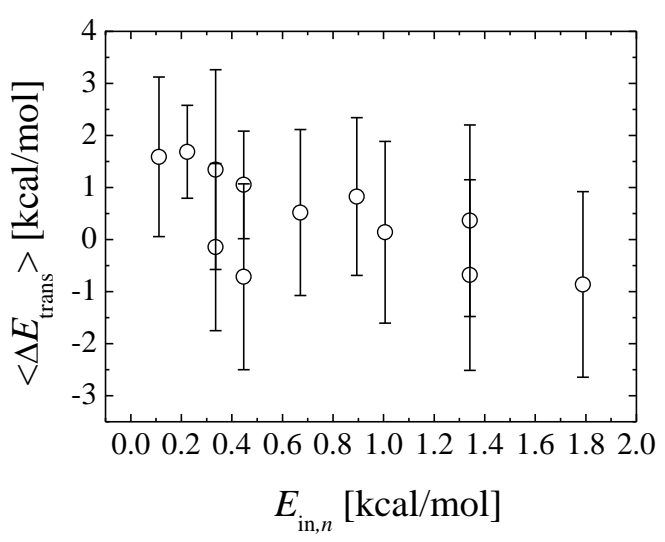

Fig.8 Average of the increase in the translational energy.

\section{Acknowledgements}

The authors would like to thank the New Energy and Industrial Technology Development Organization (NEDO) in Japan for their financial support. MD simulations were carried out using the super computer system of Institute of Fluid Science, Tohoku University.

\section{References}

[1] S. Holdcroft, "Fuel Cell Catalyst Layers: A Polymer Science Perspective", Chem. Mater., Vol. 26, pp.381-393

[2] I. Kinefuchi, J. Oyama, K. Yokoyama, N. Kubo, T. Tokumasu, Y. Matsumoto, "Direct Simulation Monte Carlo Analysis of Gas Transport in Microporous Structure Based on X-Ray computed Tomography", ECS Trans., Vol.58, pp. 71-78.

[3] I. Kinefuchi, T. Terao, J. Oyama, T. Tokunaga, K. Yokoyama, N. Kubo, G. Inoue, M. Kawase, T. Tokumasu, Y. Hara, T. Hara, "Gas Transport Analysis in Catalyst Layers of PEFCs Based on the Real Material Structure", The 54th Battery Symposium in Japan.

[4] T. Mabuchi, T. Tokumasu, "Effect of Bond State of Water on Hydronium Ion Mobility in Hydrated Nafion Using Molecular Dynamics Simulation", J. Chem. Phys., Vol.141, 104904.

[5] S. S. Jang, V. Molinero, T. Cagin, and W. A. Goddard, "Nanophase-Segregation and Transport in Nafion 117 from Molecular dynamics Simulations: Effect of Monomeric Sequence", J. Chem. Phys., Vol.108, pp. 3149-3157.

[6] L. Dutkiewicz, R. E. Johnson, A. Vertes, R. Pedrys, "Molecular Dynamics Study of Vibrational Excitation Dynamics and Desorption in Solid $\mathrm{O}_{2}$ ", J. Chem. Phys., Vol.103, pp. 2925-2933.

[7] S. Banerjee, M. Liu, and R. E. Johnson, "Molecular dynamics study of low energy cascades in solids: desorption from solid molecular oxygen", Surface Science Letters, Vol.255, pp.504-508.

[8] T.Ohmori, Y.Kimura, "Anomaly of the temperature dependence of the diffusion of oxygen in supercritical water", J. Chem. Phys, Vol.116, pp.2680-2683.

[9] H. J. Castejon, C. R. Bernecker, "Modeling the liquid-gas interface using self-assembled monolayers", Surf. Sci, Vol.606, pp.1274-1279.

[10] W. A. Alexander, D. Troya, "Theoretical Study of the Dynamics of Collisions Between HCL and $\omega$-Hydroxylated Alkanthiol Self-Assembled Monolayers", J. Phys. Chem. C, Vol.115, pp.2273-2283. 